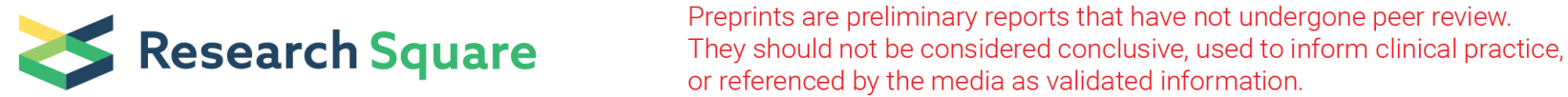

\section{LncRNA URHC promotes cell proliferation by regulating a miR-5007-3p/DNAJB9 axis in hepatocellular carcinoma}

kunwei niu

Xijing hospital

Shibin Qu

xijing hosptial

Xuan Zhang

Xijing Hospital

Jimin Dai

Xijing hospital

Jianlin Wang

Xijing hospital

Ye Nie

Xijing hospital

Hong Zhang

Xijing hospital

Kaishan Tao

Xijing Hospital

Wenjie Song ( $\nabla$ surgeon2002@163.com )

Xijing hospital

\section{Research}

Keywords: LncRNA, miR-5007-3p, DNAJB9, hepatocellular carcinoma, cell proliferation

Posted Date: November 6th, 2020

DOI: https://doi.org/10.21203/rs.3.rs-100940/v1

License: (c) (1) This work is licensed under a Creative Commons Attribution 4.0 International License.

Read Full License 


\section{Abstract}

Background: To investigate the underlying mechanisms of IncRNA URHC in HCC.

Methods: RT-qPCR, FISH staining, EdU, colony formation, and tumor xenografts experiments were used to identify localized and biological effects of URHC on HCC cells in vitro and in vivo. The Bioinformatics analysis, Dual- luciferase reporter assay, and rescue experiments revealed the potential mechanism of URHC.

Results: We found that URHC was mainly localized in the cytoplasm. URHC silencing may inhibit the HCC cells proliferation. And URHC positively regulated the level of DNAJB9 by sponging miR-5007-3p.

Conclusion: Together, our study elucidated the role of URHC as a miRNA sponge in HCC, and shed new light on IncRNA-directed diagnostics and therapeutics in HCC.

\section{Background}

Hepatocellular carcinoma (HCC) is the most common type of liver tumor worldwide. It has become the second leading cause of cancer-related death in China [1,2]. Tumor resection is currently the best treatment choice among the several therapeutic methods available. However, $\mathrm{HCC}$ is often diagnosed at a late stage, when the prognosis is poor, and many patients die within 1 year after the detection of HCC [3]. Therefore, enhancing our understanding of the molecular mechanisms in $\mathrm{HCC}$ is essential for the development of effective treatment strategies. Recent studies have shown that the regulation of noncoding RNAs (ncRNAs) plays a crucial role in HCC, which may contribute to the development of new effective therapeutic strategies to improve the prognosis of HCC patients.

NcRNAs comprise long non-coding RNAs (IncRNAs), microRNAs (miRNAs), small nuclear RNA (snRNA), piwi-interacting (piRNA), and so on. LncRNAs are a class of conserved non-protein-coding RNAs with more than 200 nucleotides that are widely distributed in the human genome [4, 5]. They can regulate gene expression in cis or in trans by diverse mechanisms [6-8], and have been demonstrated to control a variety of biological processes, such as proliferation, apoptosis, migration, invasion, tumorigenicity, and metastasis, by interacting with DNA, RNA, and proteins $[9,10]$. miRNAs are defined as small ncRNAs with approximately 19-25 nucleotides that modulate gene expression via binding to the 3 '-untranslated region (3'-UTR) of their target mRNAs, leading to translation inhibition or degradation [11, 12]. By negatively regulating the expression of target genes, miRNAs play a significant role at the posttranscriptional level and participate in a large number of biological processes, including cell proliferation, cell cycle, apoptosis, and differentiation [13-15]. Recent studies have revealed that IncRNAs can function as a competing endogenous RNAs (ceRNAs) to regulate the expression pattern and biological characteristics of miRNAs $[16,17]$. snRNAs are short RNAs that are less than 350 nts and are associated with proteins to form small nuclear ribonucleoprotein particles (snRNPs) [18]. piRNAs are a germline specific class of small regulatory RNAs that repress transposons to maintain genome integrity [19]. 
IncRNAs upregulated in hepatocellular carcinoma (URHC) are located on the forward strand of human chromosome 2: 173958088-173958307, and its transcript length is $219 \mathrm{bp}$. Significantly, URHC has been found to be upregulated in HCC. Loss-of-function experiments have shown that URHC inhibitits cell proliferation, and cell cycle progression in $\mathrm{HCC}$ cells, suggesting that targeting URHC may be a potential diagnostic and therapeutic method for HCC [20]. However, the precise regulatory mechanisms of URHC signaling in HCC remain largely unknown. The expression of miR-5007-3p has been demonstrated to be upregulated in human gastric cancer [21]. However, miR-5007-3p expression in HCC is unclear. Furthermore, whether the important roles of URHC in $\mathrm{HCC}$ are associated with the dysregulation of miR5007-3p remain unknown. DnaJ homolog subfamily B member 9 (DNAJB9), also known as MDJ-1 and ERDJ4, is a member of the DNAJ protein family [22]. It has been reported that DNAJB9 suppresses cell death induced by ER stress in SK-N-SH cells [23], but the cellular function of this DNAJB9 protein is not well understood.

In the present study, we investigated the biological effects of URHC overexpression on cell proliferation in $\mathrm{HCC}$. Bioinformatics prediction and experimental analysis confirmed that URHC directly targeted miR5007-3p to regulate its expression. Furthermore, mechanistic analysis revealed that URHC positively regulated DNAJB9 by sponging miR-5007-3p, indicating that it plays an oncogenic role in HCC pathogenesis. Together, these results suggest that the URHC-miR-5007-3p-DNAJB9 axis might be a promising therapeutic target for HCC.

\section{Methods}

\section{Cell culture and transfection}

The human HCC cell line Hep3B and 293T cells were purchased from ATCC. The normal human liver cell line MIHA was purchased from YaJi Biological (Shanghai, China). HCC cells Bel-7402 and Bel-7404 were obtained from Beina Biological (Beijing, China) in 2017. The SMMC-7721 was bought from Fenghui Biotechnologies Inc (Hunan, China). MIHA, SMMC-7721, Bel-7402, and Bel-7404 cells were cultured in RPMI 1640 (Invitrogen, Carlsbad, CA, USA) containing 10\% fetal bovine serum (FBS, Invitrogen). Hep3B and 293T were cultured in Dulbecco's modified Eagle medium (DMEM, Invitrogen) containing 10\% FBS. All cells were incubated in a humidified atmosphere of $5 \% \mathrm{CO}_{2}$ at $37^{\circ} \mathrm{C}$. Dimethyl sulfoxide was purchased from Sigma-Aldrich (St. Louis, MO).

Scrambled siRNA of URHC (siRNA-Con) and URHC siRNAs were purchased from GenePharma (Shanghai, China). miR-5007-3p inhibitor, inhibitor negative control (NC inhibitor), miR-5007-3p mimic and NC mimic were also purchased from GenePharma (Shanghai, China). Full-length URHC cDNA was subcloned into GV230 lentiviruses (Genechem, Shanghai, China) and infected into Bel-7404 and Hep3B cells to generate URHC-overexpressing cells. Lipofectamine 2000 Reagents (Invitrogen Co, USA) were used for cell transfections.

\section{Human tissue samples}


We obtained 26 pairs of primary HCC and adjacent non-tumor tissues from patients undergoing surgery at Xijing Hospital, the Fourth Military Medical University; all diagnoses were based on a biopsy. These tissues were immediately frozen in liquid nitrogen after surgical resection. All patients provided written informed consent, and these studies were approved by the Ethics Review Committees of Xijing Hospital.

\section{Reverse transcription and quantitative real-time PCR.}

Total RNA was prepared from the indicated cells using TRIzol reagent (Invitrogen) based on the manufacturer's instructions. One microgram of total RNA was converted to cDNA using PrimeScript ${ }^{\circledR}$ RT Master Mix Perfect Real-Time (Takara, Inc). SYBR Premix EX Taq II (TaKaRa) was used based on the manufacturer's instructions under the following conditions: $95^{\circ} \mathrm{C}$ for $2 \mathrm{~min}, 95^{\circ} \mathrm{C}$ for $15 \mathrm{~s}$, and $60^{\circ} \mathrm{C}$ for $60 \mathrm{~s}$ for 40 cycles. miR-5007-3p expression levels were quantified using MicroRNA First-Strand Synthesis

and miRNA Quantitation Kits including U6 control primer (Takara) based on the manufacturer's instructions. The results were analyzed based on the $2-\triangle \Delta \mathrm{Cq}$ formula. The primers used for RT-qPCR were as follows: b-actin: sense 5'-CTGGAACGGTGAAGGTGACA-3', and antisense 5'-

CGGCCACATTGTGAACTTTG-3'; URHC: sense 5'- TGTTTATGTGAGAGGAGAAAGGAAG-3'; and antisense 5'CACTAGAGGTCTGCAAATAAAGTGA-3'; DNAJB9: sense 5'- TAGGCACACACCACCACATC-3'; and antisense 5'- CTTTGGGAGGCCAAGGTAGG-3'; and miR-5007-3p 5'-CCATATGAACCAAACTCTAATA-3'. The internal

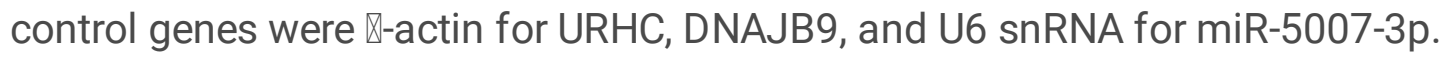

\section{Fluorescence in situ hybridization}

SMMC-7721 and Hep3B cells were plated to achieve $70 \%$ confluency for staining. Frozen sections of HCC tissues were treated with $4 \%$ DNase/RNase-free paraformaldehyde. After fixation, the sections were treated with Proteinase $\mathrm{K}(20 \mathrm{gg} / \mathrm{ml})$ for $5 \mathrm{~min}$, and then washed three times with phosphate-buffered saline (PBS). The cells were incubated with a prehybridization solution for $1 \mathrm{~h}$ at $37^{\circ} \mathrm{C}$. Then, the prehybridization solution was removed, and the cells were covered with a URHC probe $(8 \mathrm{ng} / \mu \mathrm{l})$ hybridization solution overnight in a $37^{\circ} \mathrm{C}$ incubator. The slices were washed three times using Wash Buffer Solution. The cells were extensively washed three times using Wash Buffer Solution, counterstained with DAPI, and mounted with an anti-fade reagent (Invitrogen). The URHC probe for FISH was: 5'-GFP-AGTACATACTCACTACACTAGAGGTCTGCA-GFP-3' (Servicebio, China). The mir-5007-3p probe for FISH was: 5'-Cy3-ATTAGAGTTTGGTTCATATGAT-Cy3-3' (Servicebio).

\section{CCK-8 and colony formation assays}

The CCK-8 assay was used to assess HCC cell proliferation. HCC cells were seeded in a 96-well plate at a density of $2 \times 10^{3}$ cells per well. Cell proliferation was measured at $24,48,72$, and $96 \mathrm{~h}$. CCK-8 solution $(10 \mathrm{~mL})$ was added to each well, and incubated for $1 \mathrm{~h}$ at $37^{\circ} \mathrm{C}$, in a $5 \% \mathrm{CO}_{2}$ incubator. After incubation, the optical density (OD) 450 value was read using an Epoch Spetrophotometer (USA). Experiments were repeated at least three times. 
The colony formation assay was performed to measure the capacity of cell proliferation. SMMC-7721 and Hep3B cells (300 cells/well) were seeded in a six-well plate and cultured for 10 days. Colonies were then fixed with methanol for $15 \mathrm{~min}$ and stained for $10 \mathrm{~min}$ with $0.5 \%$ crystal violet. Then, colonies were imaged with a Phone and analyzed by ImageJ software. The assays were repeated at least three times.

\section{5-Ethynyl-2'-deoxyuridine (EdU) assay}

Cell proliferation was also determined by the 5-ethynyl-2'-deoxyuridine assay using an Apollo®488 EdU Kit (RIBOBIO, Guangzhou, China). The EdU assay was performed based on the manufacturer's instructions. The cells were then visualized under a fluorescence microscope $(20 \times 10)$. To assess cell proliferation, the ratio of EdU-stained cells (with red fluorescence) to Hoechst-stained cells (with blue fluorescence) was calculated. Experiments were repeated at least three times.

\section{Luciferase reporter assay}

The luciferase reporter assay was performed in 293T cells. The 293T cells were seeded in a six-well plate and cotransfected with luciferase reporter constructs encoding the wild-type 3'-UTR region of DNAJB9 or URHC (DNAJB9/URHC-WT-3'-UTR) or a mutated 3'-UTR region of DNAJB9 or URHC 3'-UTR region (DNAJB9/URHC-MUT-3'-UTR) (RIBOBIO, Guangzhou, P.R. China) and miR-5007-3p mimic or mi-NC using Lipofectamine 2000 (Invitrogen). After $48 \mathrm{~h}$ of incubation, the cells were washed with PBS and lysed with Passive Lysis Buffer (Promega). Firefly and Renilla luciferase activities were measured using the DualLuciferase Reporter Assay Kit (Promega) based on the manufacturer's protocol.

\section{Western blot assay}

Cells were lysed using RAPI buffer (Beytime, China). Total proteins were quantified using the BCA Protein Assay Kit (Beytime, China). Proteins were separated by $12 \%$ SDS-polyacrylamide gel electrophoresis and transferred to a polyvinylidene fluoride (PVDF) membranes (Milipore, Inc). The membranes were washed three times for 10 mins with Tris-buffered saline-0.5\% Tween 20 (TBS-T) and blocked with $5 \%$ nonfat milk-TBS-T at room temperature for $1 \mathrm{~h}$. Subsequently, they were incubated with primary antibodies (antiDNAJB9 and anti-GAPDH obtained from Abcam and Cell Signaling Technology, respectively) at $4^{\circ} \mathrm{C}$ overnight. Following, incubation with corresponding secondary antibodies, the signal were detected with ECL Western Blotting Detection Reagents (Milipore, Inc). GAPDH was used as an endogenous reference.

\section{Immunohistochemical (IHC) assay}

DNAJB9 and Ki67 were detected in xenograft tumor specimens of nude mice, which were fixed with paraformaldehyde, embedded with paraffin and cut into 4 Im sections. The subsequent steps accomplished with the biotin-streptavidin peroxidase method (SPlink Detection Kit, ZSGB-Bio, Beijing, China) were followed based on the manufacturer's instructions. Briefly, paraffin-embedded samples were deparaffinized, dehydrated in a graded series of ethanol and blocked with endogenous peroxidase for $10 \mathrm{~min}$, and incubated in goat serum for $30 \mathrm{~min}$. The slides were incubated with the corresponding primary antibodies at $4^{\circ} \mathrm{C}$ overnight. Then, the slides were washed with PBS, incubated with biotinylated 
goat anti-rabbit IgG, and then incubated with horseradish peroxidase (HRP) conjugated streptomycin. Diaminobenzidine (ZSGB-Bio) was added to the slides for chromogenic reaction. The slides were observed with an optical microscope (Olympus, Tokyo, Japan).

\section{In vivo proliferation assay}

Six week-old male BALB/c nude mice were obtained from Vital River Laboratory Animal Technology Co. (Beijing, China). Animal studies were approved by the Institutional Animal Care and Use Committee of Fourth Military Medical University. The mice were housed in pathogen-free conditions. To evaluate the in vivo tumorigenic effects, mice were randomly divided into the following two groups with five mice in each group: the control group and the LncRNA URHC group. siRNA-URHC and URHC-NC cells $\left(1 \times 10^{7}\right)$ were inoculated subcutaneously in the right flank of nude mice. Tumors were measured every 7 days, and tumor volumes were calculated. Five weeks after HCC cell inoculation, the mice were sacrificed, and the tumors were collected.

\section{Statistical analysis}

All experimental data were reported as means \pm experimental standard deviations (SD). Student's t-test was used to determine the significance of the results (significance: ${ }^{\star} P<0.05 ; \star \star P<0.01,{ }^{\star \star \star} P<0.001$ ).

\section{Results}

\section{URHC is predominantly localized in the cell cytoplasm}

Since cellular location is known to dictate the function of IncRNAs, a FISH assay was performed to identify the subcellular localization of URHC in 7721 cells, Hep3B cells, and HCC tissues. Moreover, cellular fractionation was performed to identify the subcellular localization of URHC in 7721 and Hep3B cells. The results confirmed that URHC was mainly localized in the cell cytoplasm (Figure 1A, B, and C).

\section{MiR-5007-3p is a target of URHC in HCC cells}

Previous data indicate that URHC expression is upregulated in HCC cell lines and tissues [20]. 7404 and Hep3B cells exhibited lower URHC expression than the 7721 and $7402 \mathrm{HCC}$ cell lines (Figure 2A), so we selected 7404 and Hep3B cells to construct stable cell lines to overexpress URHC, and the transfection efficiency was detected by RT-qPCR (Figure 2B). Increasing evidence has revealed that IncRNAs contain sequences that are complementary to miRNAs and that they can directly or indirectly regulate the expression and activity of miRNAs. The potential targets of URHC were predicted by the bioinformatics database (StarBase). We observed that several potential miRNAs had putative binding sites for URHC, such as miR-5007-3p, miR-4698, miR-559, and miR-3942-3p (Table 1). Compared with the other three potential miRNAs, the expression level of miR-5007-3p was the lowest in the URHC-high expressing of HCC cells and highest in the URHC-low expressing HCC cells (Figure 2C and Figure S1). To confirm our prediction that miR-5007-3p targets to URHC, we first measured the expression levels of miR-5007-3p in 
7404 and Hep3B cells that were transfected with over-URHC or siRNA-URHC, respectively, by RT-qPCR. The results showed that the expression of miR-5007-3p was decreased in the over-URHC group compared with the over-control group, whereas the expression of miR-5007-3p was increased in the siRNA-URHC group compared with the siRNA-control group (Figure 2D). Next, we measured the expression of miR-5007-3p in HCC tissues and normal tissues. As shown in the RT-qPCR results in Fig 2E, the expression of miR-5007$3 p$ was significantly downregulated in HCC tissues compared with that in the adjacent tissues.

Additionally, we determined the location of URHC and miRNA-5007-3p in HCC tissues, normal tissues, and cell lines. The FISH results showed that URHC and miRNA-5007-3p were colocalized in the cell cytoplasm (Figure 2F). These results suggest that URHC may negatively regulate the expression of miR-5007-3p. Moreover, we confirmed the direct relationship between URHC and miR-5007-3p using a dual luciferase reporter assay. Using the bioinformatic database, we predicted the potential miRNA binding sites in URHC, and the alignment of miR-5007-3p with the 3'-UTR of URHC was shown (Figure 2G). 293T cells were cotransfected with miRNAs and URHC (URHC-NC, URHC mutant). As shown in Fig. 2H, miR-5007-3p mimic transfection significantly decreased the luciferase activities of URHC compared with NC transfection, whereas there were no effects on the luciferase activities by miR-5007-3p mutant transfection. These results indicate that URHC directly targets miR-5007-3p to regulate its expression.

\section{The expression of DNAJB9 is regulated by URHC \& has-miR-5007-3p in HCC cells}

It is well known that miRNAs play biological roles by targeting the 3'-UTR of target genes. The bioinformatics databasses (Targetscan, miRDB, miRTarBase and miRWalk) predicted that potential downstream genes of miR-5007-3p include BCOR, AJAP1, DNAJB9, RAI1, and others (Table 2). Among the targets of miR-5007-3p, we focused on DNAJB9, because the expression level of DNAJB9 was the highest in the HCC cells that highly expressed URHC (Figure S2). We first measured the expression of DNAJB9 in HCC tissues, and its expression was found to be significantly increased (Figure $3 A$ ). Furthermore, the URHC and DNAJB9 expression showed a significant positive correlation in HCC patients by Spearman's correlation analysis, whereas miR-5007-3p and DNAJB9 expression showed a significant negative correlation (Fig 3B). In 7404 and Hep3B cell lines, the mRNA level of DNAJB9 was significantly decreased in cells transfected with siRNA-URHC compared with control cells, whereas the expression of DNAJB9 in over-URHC cells showed the opposite result (Figure $3 C$ ). Using the bioinformatic database, we predicted the potential miRNA binding sites in DNAJB9, and the alignment of miR-5007-3p with the 3'-UTR of $D N A J B 9$ was shown (Figure 3D). We then confirmed the direct interaction between miR-5007-3p and the 3'-UTR of DNAJB9 using a dual-luciferase reporter assay. 293T cells were cotransfected with miRNAs and $D N A J B 9$ (DNAJB9 NC, DNAJB9 mutant). As shown in Figure 3E, miR-5007-3p transfection significantly decreased the luciferase activities of $D N A J B 9$, compared with NC transfection, whereas there were no effects on the luciferase activities by miR-5007-3p mutant transfection. The above results suggested that URHC functioned as a ceRNA by sponging miR-5007-3p and indirectly regulated DNAJB9 expression.

\section{has-miR-5007-3p reverses the promoting effect of URHC on cell growth}


The previous results of CCK-8 and EdU assays demonstrated that the downregulated expression of URHC attenuated the proliferation of 7721 cells ${ }^{20}$, whereas the overexpression of URHC enhanced 7404 , and Hep3B cells proliferation by CCK-8 and colony formation assays (Figure 4A and B). We next determined whether URHC exerted its function through miR-5007-3p in 7721 and Hep3B cells In EdU and colony formation assays, the proliferation of 7721 cells in the siRNA-URHC + miR-5007-3p inhibitor group was remarkably increased compared with that in the siRNA-URHC and siRNA-URHC significant + miR-5007-3p inhibitor NC groups, whereas, HCC cell proliferation was evidently decreased in the over-URHC + miR5007-3p mimic group compared with the over-URHC and over-URHC + miR-5007-3p mimic NC groups (Figure 4C and D). Furthermore, we determined whether miR-5007-3p was involved in the URHC regulation $D N A J B 9$ expression. We assessed the effects of URHC and miR-5007-3p on the mRNA and protein levels of $D N A J B 9$ by RT-qPCR and western blot, and found that $D N A J B 9$ expression levels were apparently affected by URHC and miR-5007-3p. As shown in Figure 4E and F, DNAJB9 expression was significantly decreased after transfecting with siRNA-URHC and miR-5007-3p inhibitor NC. The inhibitory effect of siRNA-URHC was notably reversed by cotransfection with siRNA-URHC and miR-5007-3p inhibitor in 7721 cells. Otherwise, the expression of DNAJB9 was significantly increased in the over-URHC and miR-5007$3 p$ mimic NC groups. URHC advance was also reversed by transfection with miR-5007-3p mimic in Hep3B cells. These results illustrated that miR-5007-3p played a crucial role in the effect of URHC on cell proliferation. Moreover, miR-5007-3p was involved in the URHC regulation of DNAJB9 expression.

\section{URHC silencing suppressed HCC tumor growth in vivo}

To further determine the anti-tumorigenesis potential of URHC in vivo, 7721 cells transfected with siRNA$\mathrm{NC}$ and URHC-siRNA were inoculated into male nude mice. Mice were randomly divided into two groups with five mice in each group, but each group only included three tumors. Mice injected with siRNA-URHC 7721 cells showed a reduction in tumor volume and weight at the end of the experiment compared with the control groups (Figure 5A, B, and C). Moreover, IHC staining revealed that Ki67 expression was decreased in the siRNA-URHC xenograft tumor tissues (Figure 5D). Furthermore, histologic analysis of resected tumor tissues demonstrated that URHC expression was negatively associated with miR-5007-3p and positively correlated with $D N A J B 9$ (Figure $5 \mathrm{E}, \mathrm{F}, \mathrm{G}$, and $\mathrm{H}$ ). These results further confirmed the role of $\mathrm{URHC}$ in $\mathrm{HCC}$ proliferation and provided more validation for the targeting of URHC as a therapeutic in HCC treatment.

\section{Discussion}

Recent experimental studies provide strong evidence that IncRNAs play an important role in cancer progression, and the abnormal expression of IncRNAs has been found in the HCC [24-27]. For example, IncRNA HOST2 can activate the JAK2-STAT3 signaling pathway promote epithelial-mesenchymal transition, proliferation, invasion and migration of HCC cells ${ }^{27}$. A IncRNA PVT1/miR-150/HIG2 axis regulates the proliferation, invasion, and balance of iron metabolism in HCC [28]. LncRNAs have been shown to function as miRNA sponges, which interact with miRNAs and play crucial roles in human 
carcinogenesis by modulating the expression of miRNA target genes [29-32]. In this study, we found that URHC functioned as a ceRNA by sponging miRNA-5007-3p to modulate DNAJB9 expression.

A previous study showed that URHC was highly expressed in HCC cell lines and tissues [20]. However, the precise regulatory mechanisms of URHC signaling in HCC are still largely unknown. A study has shown that miR-5007-3p is expressed in the lymphatic metastasis tissue from gastric cancer patients via RTqPCR. In addition, the results revealed that expressed of miR-5007-3p was expressed in patients with positive lymphatic metastasis of primary gastric tumors [21], but miR-5007-3p expression in HCC is unclear. Whether the important roles of URHC in HCC are associated with the dysregulation of miR-5007$3 p$ also remains unknown. DNAJB9 also known as MDJ-1 and ERDJ4, is a 223-amino acid protein that is a member of the DNAJ protein family. It is expressed in most cells and displays cytoplasmic immunoreactivity via IHC. It has been reported that $D N A J B 9$ is involved in ER stress and the unfolded protein response (UPR). It also regulates URP as a Bip/Grp78 cochaperone. Meanwhile, DNAJB9 influences many cellular processes by regulating the ATPase activity of $70 \mathrm{kD}$ heat shock proteins [22, 33]. However, the cellular function of this DNAJB9 remains largely unknown.

In this study, we investigated the expression pattern of URHC in HCC. The results confirmed that URHC was preferentially localized in the cell cytoplasm, suggesting that it could function as a ceRNA to regulate the expression pattern and biological characteristics of miRNAs. In the present study, we found that an inverse correlation between URHC and miR-5007-3p expression. URHC bound directly to miR-5007-3p, thereby controlling miR-5007-3p availability for its target gene DNAJB9. Furthermore, we confirmed that $D N A J B 9$ was highly associated with URHC. Signficantly, miR-5007-3p could reverse the promoting effect of URHC on cell proliferation. in vivo xenograft tumor assays, URHC silencing reduced the tumor volume and weight. The results showed that URHC positively regulated the level of DNAJB9 by sponging miR5007-3p, and that it played an oncogenic role in HCC progression. Taken together, these data suggested that URHC might play an oncogenic role by regulating a URHC/miR-5007-3p/DNAJB9 axis.

\section{Conclusions}

In summary, URHC promoted HCC cell proliferation in vitro and in vivo, suggesting that URHC exhibited oncogenic properties in HCC progression. URHC promoted proliferation via sponing miR-5007-3p form $D N A J B 9$ both in vitro and in vivo. This URHC/miR-5007-3p/DNAJB9 regulatory network may shed light on tumorigenesis in $\mathrm{HCC}$ and may be contribute to the development of novel diagnostic and therapeutic methods for HCC.

\section{Declarations}

\section{Acknowledgements}

The authors thank for anonymous reviewers' constructive comments and suggestions.

\section{Authors' contributions}


Kunwei Niu. Wenjie Song and Kaishan Tao performed the majority of the experiments, analyzed the data, and wrote and edited the manuscript. Shibin Qu, Jimin Dai, Ye Nie, and Hong Zhang performed these experiments. Xuan Zhang and Jianlin Wang performed the statistical analyses. All authors reviewed the manuscript.

\section{Funding}

This study was funded by the National Nature Science Foundation of China, Grant/Award number: 81672716; the Youth Program of National Natural Science Foundation of China, Grant/Award number: 81900571.

\section{Availability of data and materials}

All of the data and materials are available.

\section{Ethics approval and consent to participate}

All patients provided written informed consent, and these studies were approved by the Ethics Review Committees of Xijing Hospital, Fourth Military Medical University [grant no: KY20183235-1]. Animal studies were approved by the Institutional Animal Care and Use Committee of Fourth Military Medical University [grant no: KY20191025-1], and in accordance with the Guide for the Care and Use of Laboratory Animals by the US National Institutes of Health.

\section{Consent for publication}

All authors have given their consent for the manuscript to be published.

\section{Competing interests}

The authors declare that they have no competing interests.

\section{Author details}

${ }^{1}$ Department of Hepatobiliary Surgery, Xijing Hospital, Fourth Military Medical University, 127 Changle Road, xi'an, Shaanxi 710032, China.

\section{References}

1. Xiao S, Yang M, Yang H, et al. miR-330-5p targets SPRY2 to promote hepatocellular carcinoma progression via MAPK/ERK signaling. Oncogenesis. 2018;7(11): 90.

2. Li H, Zhang M, Linghu E et al. Epigenetic silencing of TMEM176A activates ERK signaling in human hepatocellular carcinoma. Clin Epigenetics. 2018; 10(1): 137.

3. Li S, Yao J, Xie M, et al. Exosomal miRNAs in hepatocellular carcinoma development and clinical responses. J Hematol Oncol. 2018; 11(1): 54. 
4. Guo XB, Hua Z, Li C et al. Biological significance of long non-coding RNA FTX expression in human colorectal cancer. Int J Clin Exp Med. 2015; 8(9): 15591-15600.

5. Hombach S, Kretz M. Non-coding RNAs: Classification, Biology and Functioning. Adv Exp Med Biol. 2016; 937: 3-17.

6. Quan M, Chen J, Zhang D. Exploring the secrets of long noncoding RNAs. Int J Mol Sci. 2015; 16(3): 5467-5496.

7. Dimitrova N, Zamudio JR, Jong RM et al. LincRNA-p21 activates p21 in cis to promote Polycomb target gene expression and to enforce the G1/S checkpoint. Mol Cell. 2014; 54(5): 777-790.

8. Zhu L, Liu Y, Chen Q et al. Long-Noncoding RNA Colorectal Neoplasia Differentially Expressed Gene as a Potential Target to Upregulate the Expression of IRX5 by miR-136-5P to Promote Oncogenic Properties in Hepatocellular Carcinoma. Cell Physiol Biochem. 2018; 50(6): 2229-2248.

9. Yang G, Lu X, Yuan L. LncRNA: a link between RNA and cancer. Biochim Biophys Acta. 2014; 1839(11): 1097-1109.

10. Marchese FP, Raimondi I, Huarte M. The multidimensional mechanisms of long noncoding RNA function. Genome Biol. 2017; 18(1): 206.

11. Kota J, Chivukula RR, O'Donnell KA et al. Therapeutic microRNA delivery suppresses tumorigenesis in a murine liver cancer model. Cell. 2009; 137(6): 1005-1017.

12. Hatziapostolou M, Polytarchou C, Aggelidou E et al. An HNF4a-miRNA inflammatory feedback circuit regulates hepatocellular oncogenesis. Cell. 2011; 147(6): 1233-1247.

13. Zheng B, Liang L, Wang $C$ et al. MicroRNA-148a suppresses tumor cell invasion and metastasis by downregulating ROCK1 in gastric cancer. Clin Cancer Res. 2011; 17(24): 7574-7583.

14. Mori $M$, Triboulet $R$, Mohseni $M$ et al. Hippo signaling regulates microprocessor and links cell-densitydependent miRNA biogenesis to cancer. Cell. 2014; 156(5): 893-906.

15. Zhang JG, Shi Y, Hong DF et al. MiR-148b suppresses cell proliferation and invasion in hepatocellular carcinoma by targeting WNT1/ $\beta$-catenin pathway. Sci Rep. 2015; 5: 8087.

16. Liu XH, Sun M, Nie FQ et al. LncRNA HOTAIR functions as a competing endogenous RNA to regulate HER2 expression by sponging miR-331-3p in gastric cancer. Mol Cancer. 2014; 13: 92.

17. Qu J, Li M, Zhong W, Hu C. Competing endogenous RNA in cancer: a new pattern of gene expression regulation. Int J Clin Exp Med. 2015; 8(10): 17110-17116.

18. Guiro J, Murphy S. Regulation of expression of human RNA polymerase II-transcribed snRNA genes. Open Biol. 2017; 7(6): 170073.

19. Sato K, Siomi MC. The piRNA pathway in Drosophila ovarian germ and somatic cells. Proc Jpn Acad Ser B Phys Biol Sci. 2020; 96(1): 32-42.

20. Xu WH, Zhang JB, Dang $Z$ et al. Long non-coding RNA URHC regulates cell proliferation and apoptosis via ZAK through the ERK/MAPK signaling pathway in hepatocellular carcinoma. Int J Biol Sci. 2014; 10(7): 664-676. 
21. Yang $B$, Jing $C$, Wang $\mathrm{J}$ et al. Identification of microRNAs associated with lymphangiogenesis in human gastric cancer. Clin Transl Oncol. 2014; 16(4): 374-379.

22. Lee HJ, Kim JM, Kim KH, et al. Genotoxic stress/p53-induced DNAJB9 inhibits the pro-apoptotic function of p53. Cell Death Differ. 2015; 22(1): 86-95.

23. Kurisu J, Honma A, Miyajima H, et al. MDG1/ERdj4, an ER-resident DnaJ family member, suppresses cell death induced by ER stress. Genes Cells. 2003; 8(2): 189-202.

24. Zhao J, Greene CM, Gray SG, Lawless MW. Long noncoding RNAs in liver cancer: what we know in 2014. Expert Opin Ther Targets. 2014; 18(10): 1207-1218.

25. DiStefano JK. Long noncoding RNAs in the initiation, progression, and metastasis of hepatocellular carcinoma. Noncoding RNA Res. 2017; 2(3-4): 129-136.

26. He JH, Han ZP, Liu JM et al. Overexpression of Long Non-Coding RNA MEG3 Inhibits Proliferation of Hepatocellular Carcinoma Huh7 Cells via Negative Modulation of miRNA-664. J Cell Biochem. 2017; 118(11): 3713-3721.

27. Xiao JN, Yan TH, Yu RM et al. Long non-coding RNA UCA1 regulates the expression of Snail2 by miR203 to promote hepatocellular carcinoma progression. J Cancer Res Clin Oncol. 2017; 143(6): 981990.

28. Wu Y, Yuan T, Wang WW et al. Long Noncoding RNA HOST2 Promotes Epithelial-Mesenchymal Transition, Proliferation, Invasion and Migration of Hepatocellular Carcinoma Cells by Activating the JAK2-STAT3 Signaling Pathway. Cell Physiol Biochem. 2018; 51(1): 301-314.

29. Xu Y, Luo X, He W et al. Long Non-Coding RNA PVT1/miR-150/HIG2 Axis Regulates the Proliferation, Invasion and the Balance of Iron Metabolism of Hepatocellular Carcinoma. Cell Physiol Biochem. 2018; 49(4): 1403-1419.

30. Wang J, Liu X, Wu H et al. CREB up-regulates long non-coding RNA, HULC expression through interaction with microRNA-372 in liver cancer. Nucleic Acids Res. 2010; 38(16): 5366-5383.

31. Chang YN, Zhang K, Hu ZM et al. Hypoxia-regulated IncRNAs in cancer. Gene. 2016; 575(1): 1-8.

32. Li T, Meng XL, Yang WQ. Long Noncoding RNA PVT1 Acts as a "Sponge" to Inhibit microRNA-152 in Gastric Cancer Cells. Dig Dis Sci. 2017; 62(11): 3021-3028.

33. Andeen NK, Yang HY, Dai DF, et al. DnaJ Homolog Subfamily B Member 9 Is a Putative Autoantigen in Fibrillary GN. J Am Soc Nephrol. 2018; 29(1): 231-239.

\section{Supporting Information}

Additional supporting information may be found online in the Supporting Information section.

\section{Tables}

Table 1 


\begin{tabular}{lccccccc}
\hline \multicolumn{1}{c}{ Name } & Accession & $\begin{array}{c}\text { Query } \\
\text { start }\end{array}$ & $\begin{array}{c}\text { Query } \\
\text { start }\end{array}$ & $\begin{array}{c}\text { Subject } \\
\text { start }\end{array}$ & $\begin{array}{c}\text { Subjec } \\
\text { t end }\end{array}$ & Score & Evalue \\
\hline hsa-miR-5007-3p & MIMAT0021036 & 74 & 91 & 4 & 21 & 72 & 1.5 \\
hsa-miR-4698 & MIMAT0019793 & 59 & 73 & 2 & 16 & 66 & 4.7 \\
hsa-miR-559 & MIMAT0003223 & 66 & 85 & 1 & 20 & 64 & 6.9 \\
hsa-miR-3942-3p & MIMAT0019230 & 164 & 181 & 4 & 21 & 63 & 8.3 \\
\hline
\end{tabular}

\section{Table 2}

\begin{tabular}{ccccccc}
\hline miRNA & Accession & Gene & miRDB & miRTarBase & miRWalk & TargetScan \\
\hline hsa-miR-5007-3p & MIMAT0021036 & BCOR & 1 & 1 & 1 & 0 \\
hsa-miR-5007-3p & MIMAT0021036 & AJAP1 & 1 & 1 & 1 & 0 \\
hsa-miR-5007-3p & MIMAT0021036 & DNAJB9 & 1 & 1 & 1 & 0 \\
hsa-miR-5007-3p & MIMAT0021036 & RAI1 & 1 & 1 & 1 & 0 \\
hsa-miR-5007-3p & MIMAT0021036 & ZNF711 & 1 & 1 & 1 & 0 \\
hsa-miR-5007-3p & MIMAT0021036 & DDT & 1 & 1 & 1 & 0 \\
hsa-miR-5007-3p & MIMAT0021036 & WDR35 & 1 & 1 & 1 & 0 \\
\hline
\end{tabular}

\section{Figures}

A

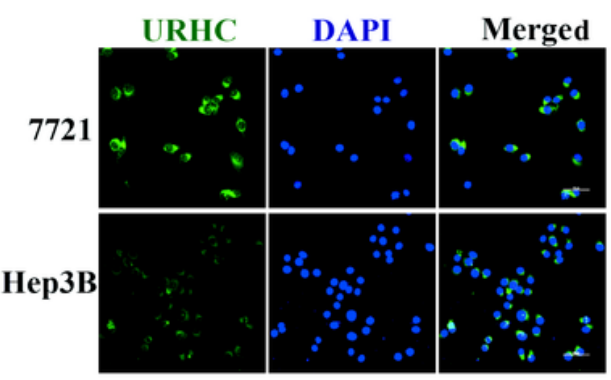

B

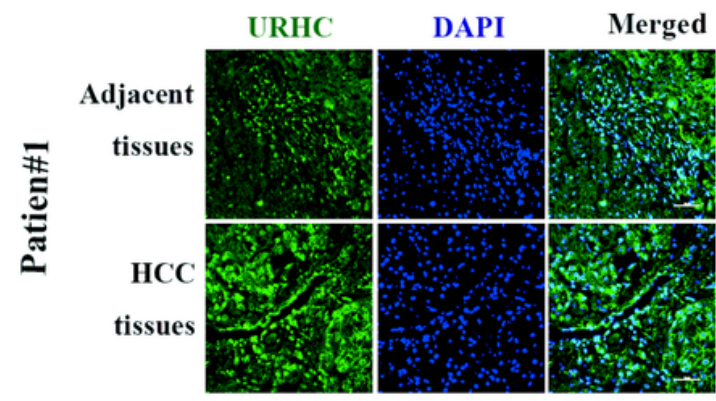

C
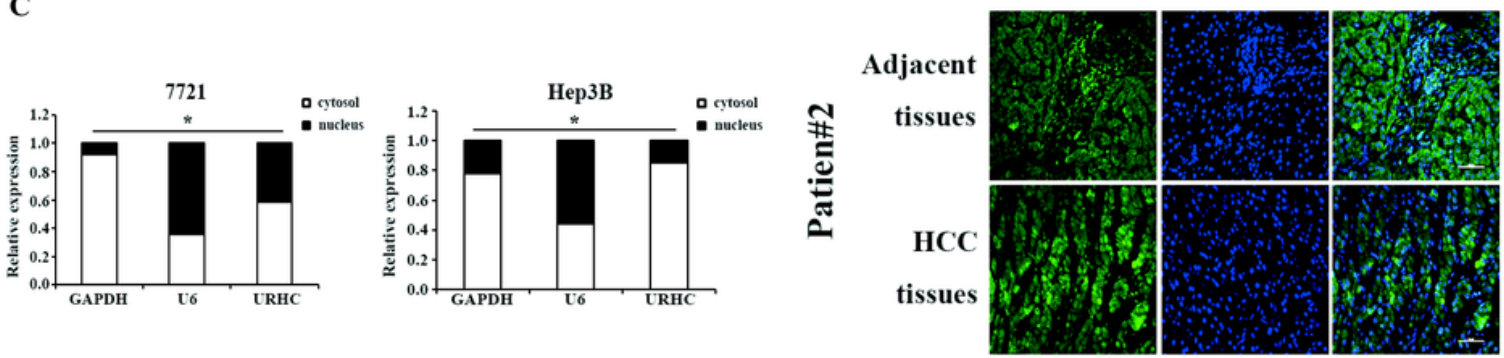

Figure 1

The results confirmed that URHC was mainly localized in the cell cytoplasm (Figure $1 \mathrm{~A}, \mathrm{~B}$, and C). 
A

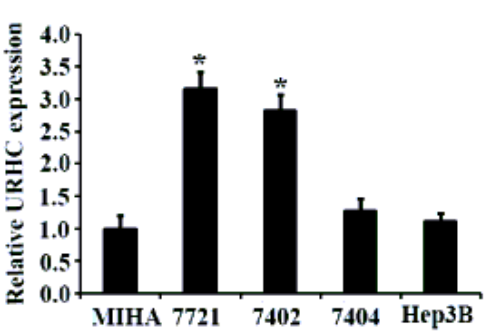

C

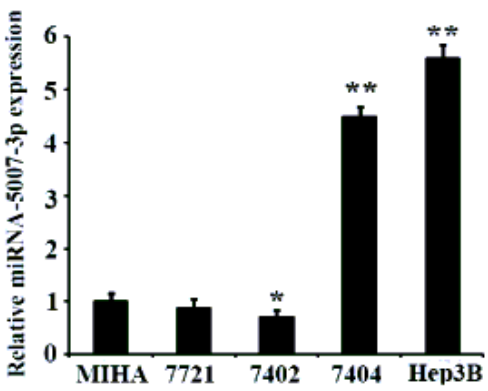

B

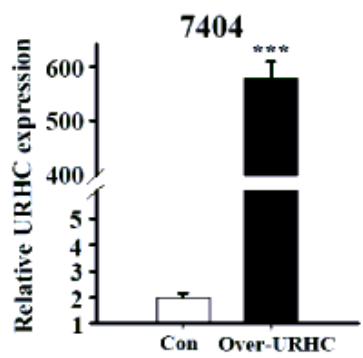

Hep3B

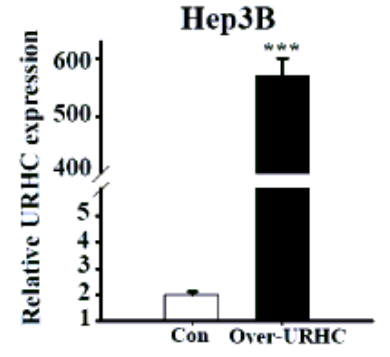

D
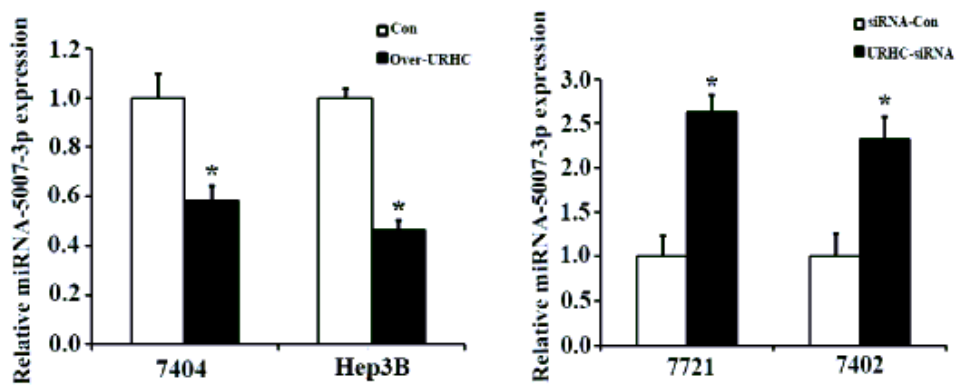

$\mathbf{F}$

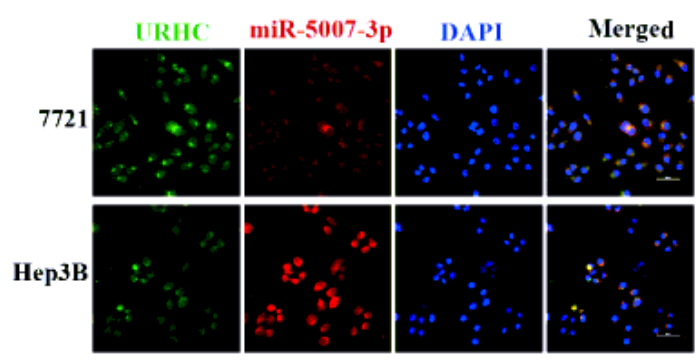

E
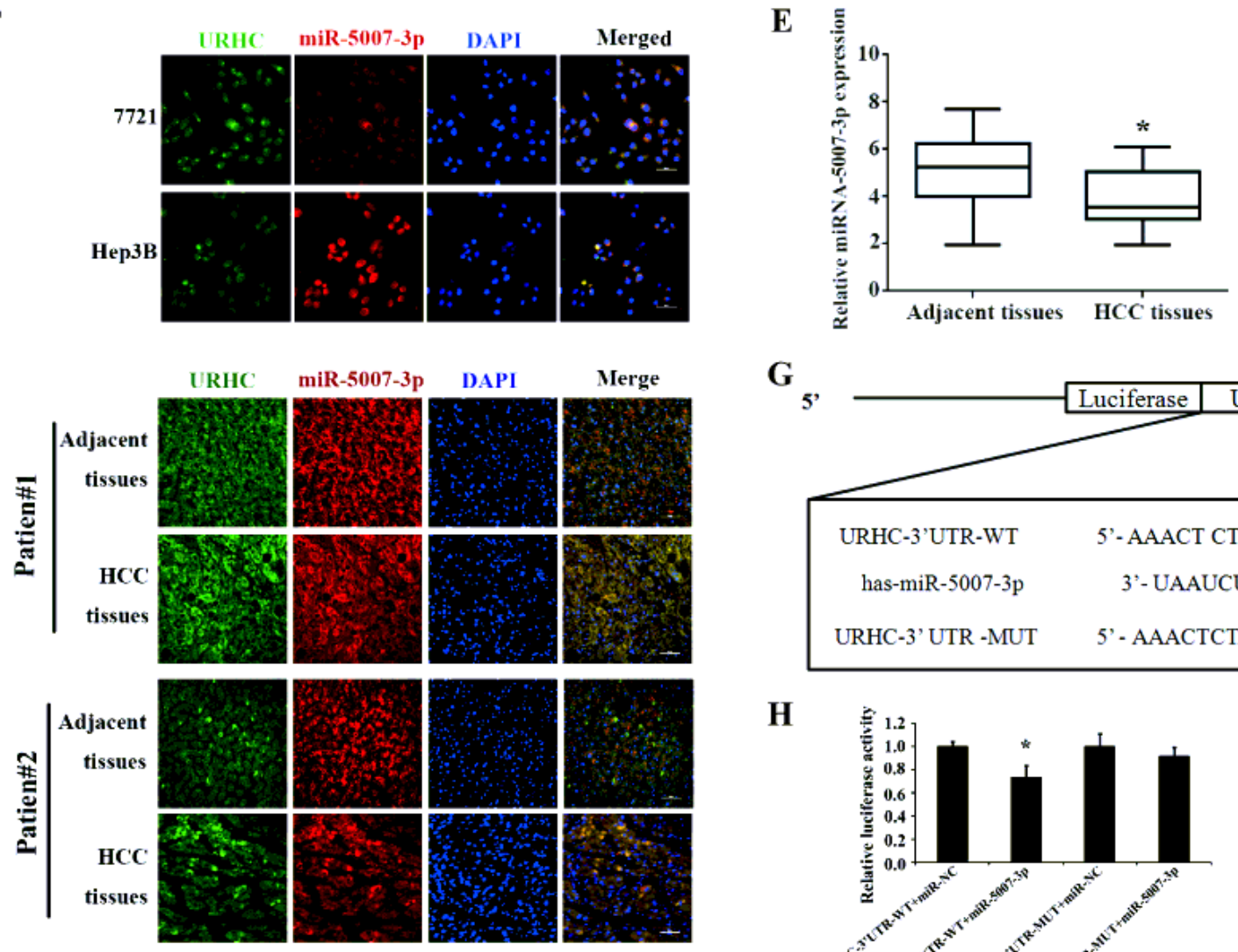

G

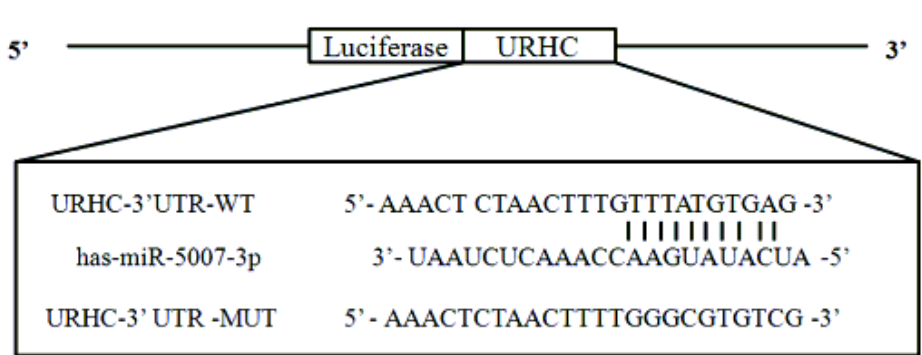

H

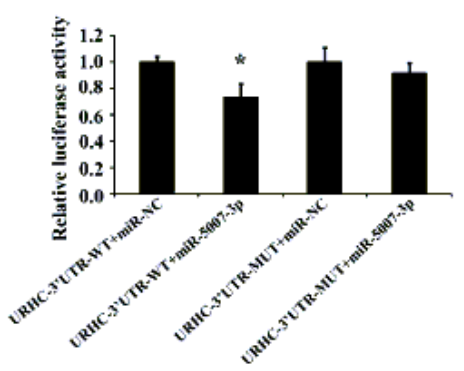

Figure 2

7404 and Hep3B cells exhibited lower URHC expression than the 7721 and 7402 HCC cell lines (Figure 2A), so we selected 7404 and Hep3B cells to construct stable cell lines to overexpress URHC, and the transfection efficiency was detected by RT-qPCR (Figure 2B). Increasing evidence has revealed that IncRNAs contain sequences that are complementary to miRNAs and that they can directly or indirectly regulate the expression and activity of miRNAs. The potential targets of URHC were predicted by the 
bioinformatics database (StarBase). We observed that several potential miRNAs had putative binding sites for URHC, such as miR-5007-3p, miR-4698, miR-559, and miR-3942-3p (Table 1). Compared with the other three potential miRNAs, the expression level of miR-5007-3p was the lowest in the URHC-high expressing of HCC cells and highest in the URHC-low expressing HCC cells (Figure 2C and Figure S1).

A

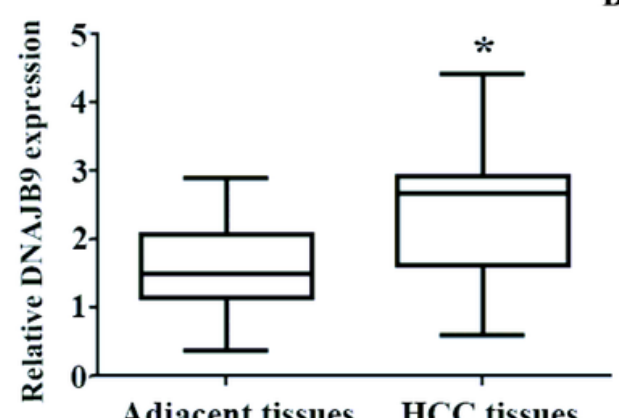

Adjacent tissues HCC tissues

C

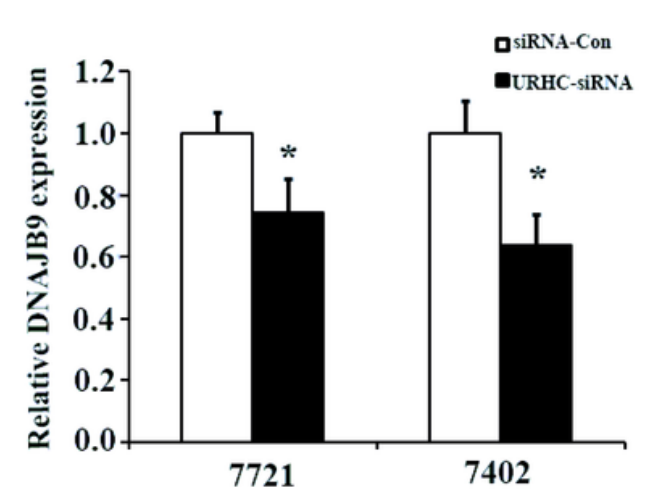

B

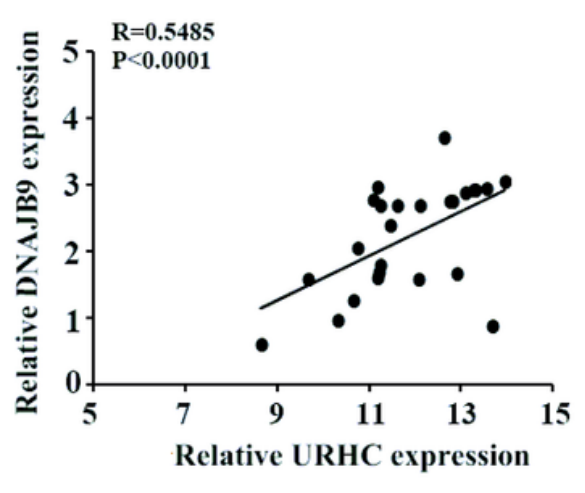

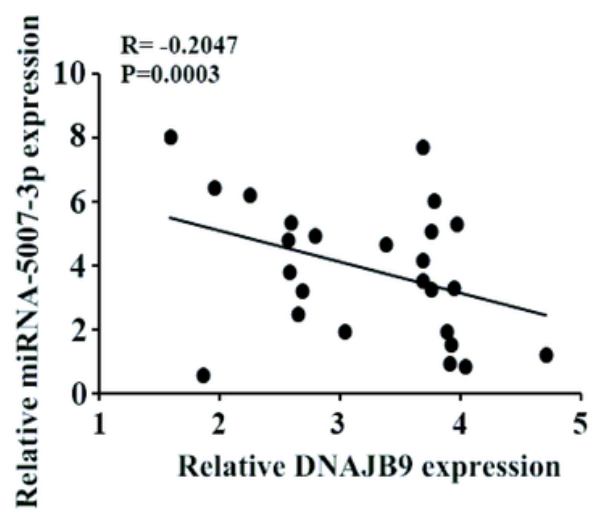

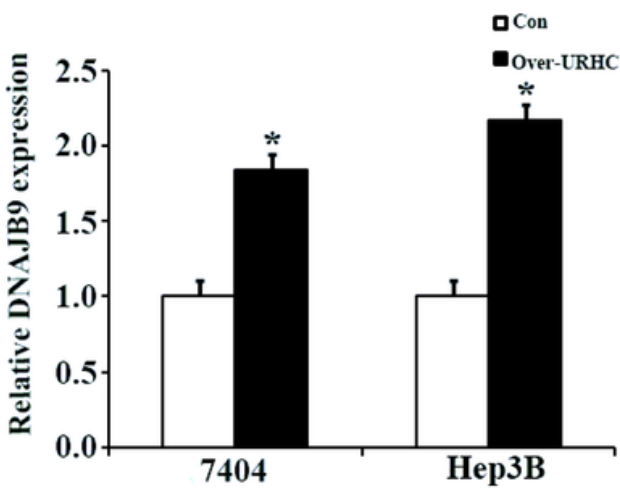

D
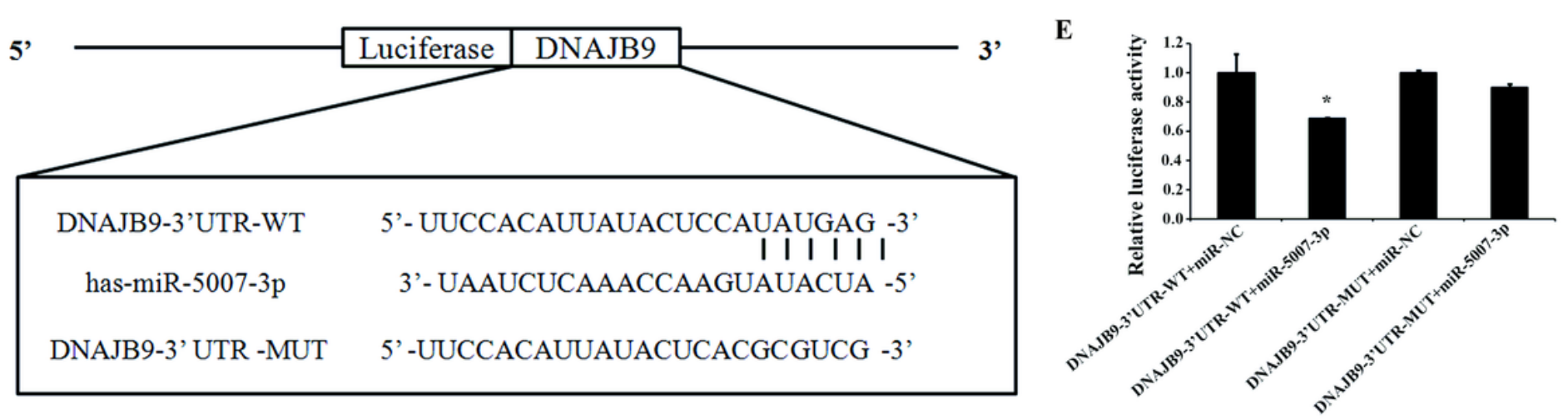

Figure 3

We first measured the expression of DNAJB9 in HCC tissues, and its expression was found to be significantly increased (Figure 3A). Furthermore, the URHC and DNAJB9 expression showed a significant positive correlation in HCC patients by Spearman's correlation analysis, whereas miR-5007-3p and DNAJB9 expression showed a significant negative correlation (Fig 3B). In 7404 and Hep3B cell lines, the mRNA level of DNAJB9 was significantly decreased in cells transfected with siRNA-URHC compared with control cells, whereas the expression of DNAJB9 in over-URHC cells showed the opposite result (Figure 3C). Using the bioinformatic database, we predicted the potential miRNA binding sites in DNAJB9, and the alignment of miR-5007-3p with the 3'-UTR of DNAJB9 was shown (Figure 3D). We then confirmed the 
direct interaction between miR-5007-3p and the 3'-UTR of DNAJB9 using a dual-luciferase reporter assay. 293T cells were cotransfected with miRNAs and DNAJB9 (DNAJB9 NC, DNAJB9 mutant). As shown in Figure 3E, miR-5007-3p transfection significantly decreased the luciferase activities of DNAJB9, compared with NC transfection, whereas there were no effects on the luciferase activities by miR-5007-3p mutant transfection. The above results suggested that URHC functioned as a ceRNA by sponging miR5007-3p and indirectly regulated DNAJB9 expression

A
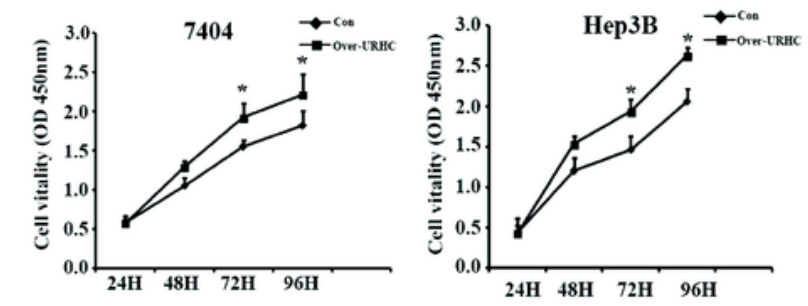

B
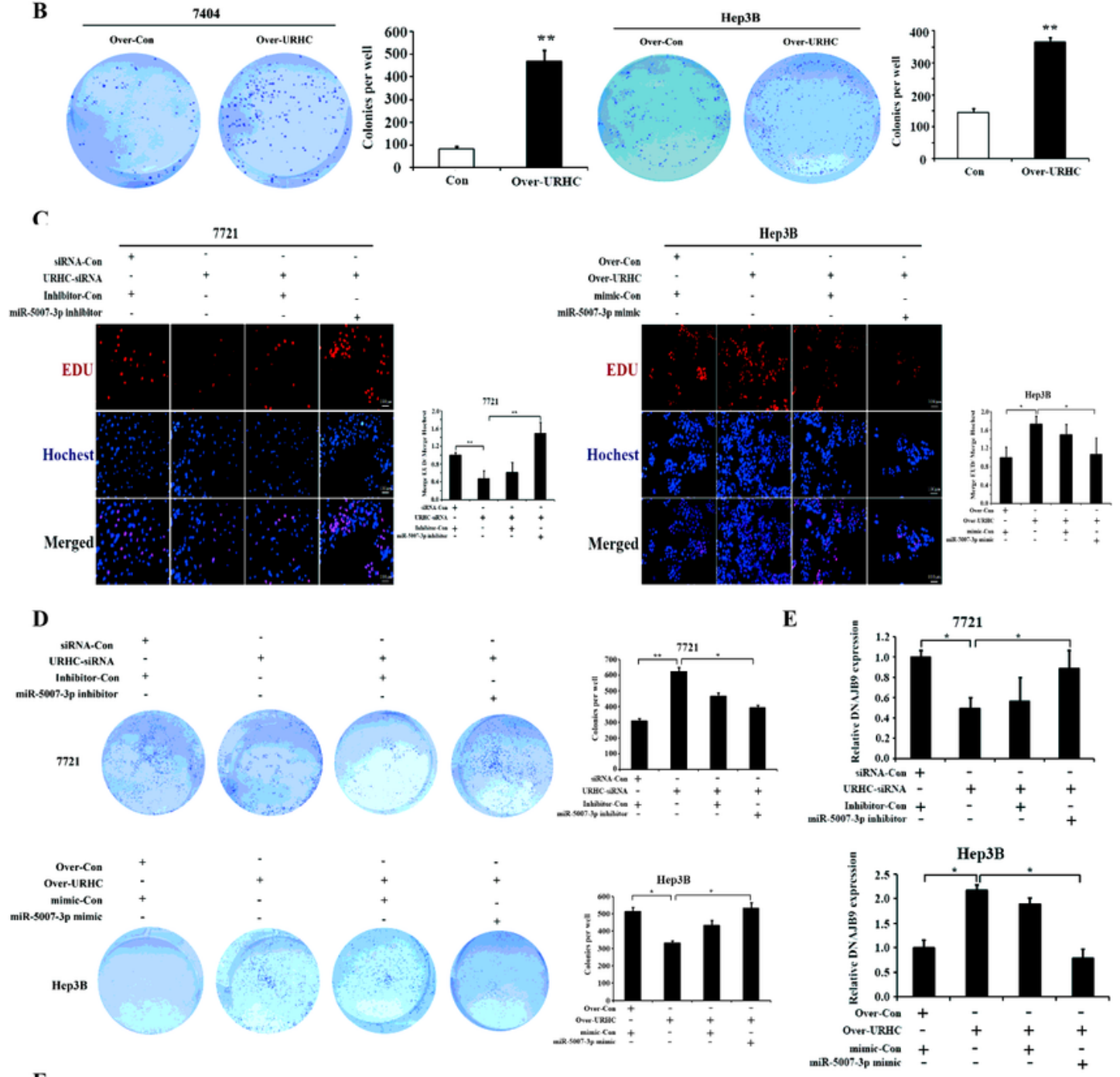

$\mathbf{F}$
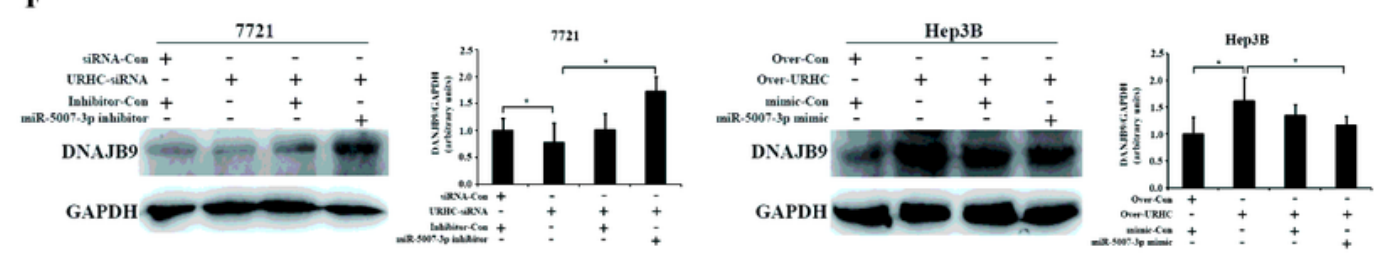

Figure 4 
The previous results of CCK-8 and EdU assays demonstrated that the downregulated expression of URHC attenuated the proliferation of 7721 cells 20 , whereas the overexpression of URHC enhanced 7404 , and Hep3B cells proliferation by CCK-8 and colony formation assays.

A
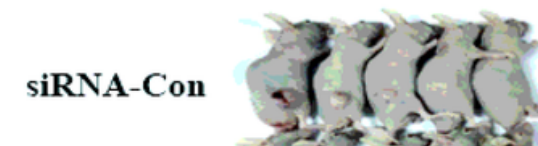

siRNA-Con

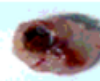

URHC-siRNA

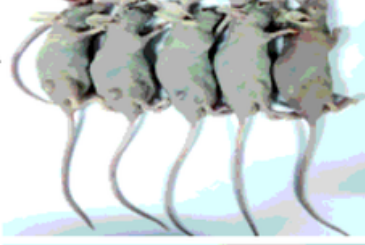

B

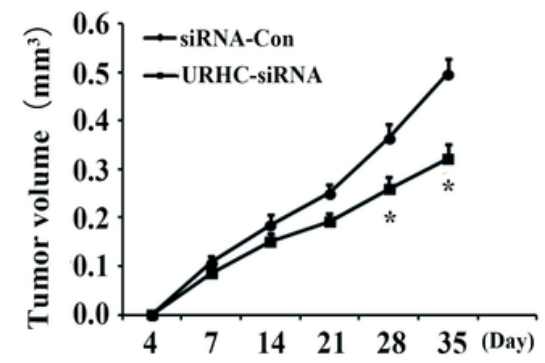

D

URHC-siRNA

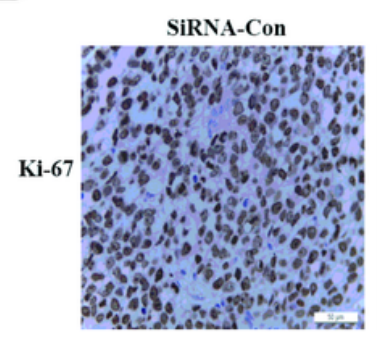

URHC-siRNA

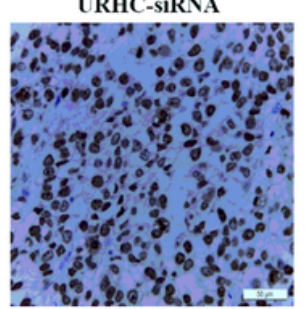

$\mathbf{E}$

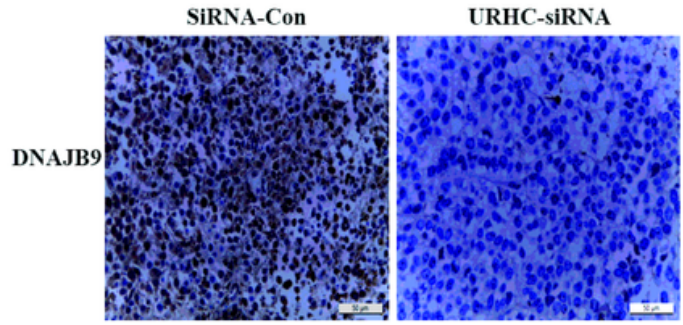

C

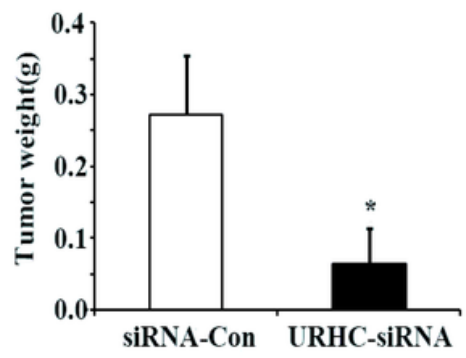

F

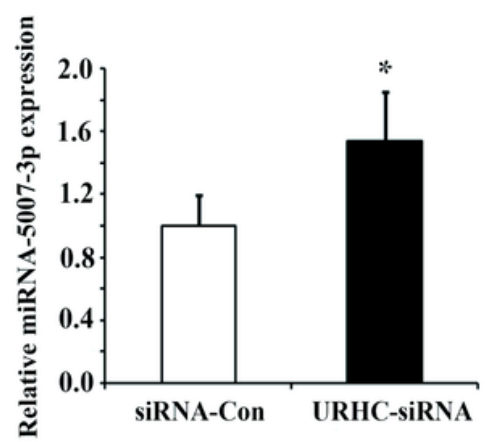

G

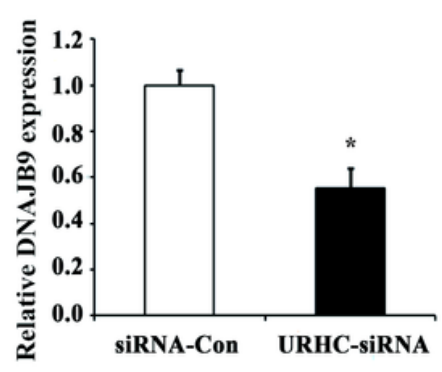

H
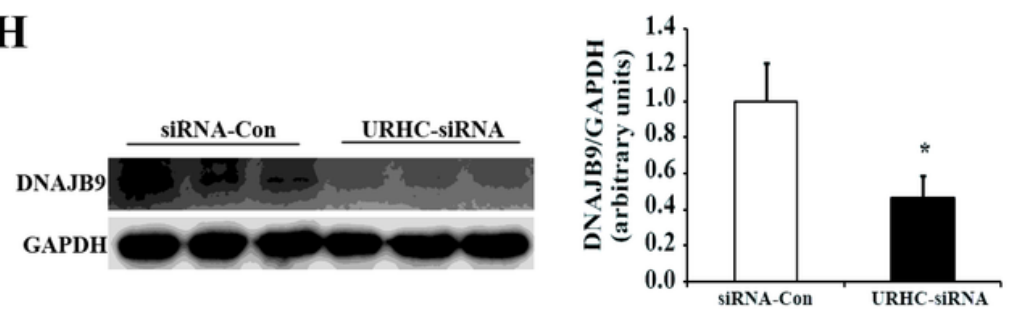

Figure 5

Mice injected with siRNA-URHC 7721 cells showed a reduction in tumor volume and weight at the end of the experiment compared with the control groups (Figure 5A, B, and C). Moreover, IHC staining revealed that Ki67 expression was decreased in the siRNA-URHC xenograft tumor tissues (Figure 5D). Furthermore, histologic analysis of resected tumor tissues demonstrated that URHC expression was negatively associated with miR-5007-3p and positively correlated with DNAJB9 (Figure 5E, F, G, and H). These results further confirmed the role of URHC in HCC proliferation and provided more validation for the targeting of URHC as a therapeutic in HCC treatment.

\section{Supplementary Files}

This is a list of supplementary files associated with this preprint. Click to download. 
- supplementaryfig.doc

Page 18/18 